\title{
Arthroscopic Debridement of Intrapelvic Abscess With Coexistent Septic Hip in an Adult Female: A Technical Note
}

\author{
Shahriar Seddigh, M.D., Alexandra Bishop, M.D., and \\ Ivan Wong, M.D., F.R.C.S.(C.), Dip.SportMed., M.A.C.M.
}

\begin{abstract}
The aim of this study was to describe the technique for debridement of an intrapelvic abscess through an arthroscopic approach. We present a 24-year-old female with an obturator internus muscle abscess that extended across the obturator membrane and into the hip joint. Decompression of the intrapelvic abscess through hip arthroscopy was planned after close examination of preoperative images. Intrapelvic space was safely accessed by hip arthroscopy. The patient was asymptomatic and had normalized biomarkers at 6 months; however, the abscess recurred at 1 year postoperation. Details of the technique for intrapelvic access and anatomic structures at risk are described in this study.
\end{abstract}

S eptic hip arthritis is a well-established orthopedic emergency with the potential for sepsis and permanent articular cartilage damage. Emergent operation with irrigation and debridement (IGD) of the hip is warranted. Open arthrotomy remains the traditional standard of treatment ${ }^{1,2}$; however, it may lead to significant morbidity and complications, including extensive soft-tissue injury, hip instability, risk of injury to the femoral head's blood supply, and subsequent avascular necrosis. Alternatively, arthroscopic hip I\&D has been used to treat septic arthritis successfully in both children and adult patients. ${ }^{3,4}$ A recent systematic review concluded that arthroscopic ISD is safe and effective in treating septic arthritis of native hips. ${ }^{5}$ Some authors have reported favorable long-term outcomes

From the Department of Orthopaedic Surgery, Nova Scotia Health Authority, Halifax, Nova Scotia, Canada (S.S., A.B.); and Division of Orthopaedic Surgery, Dalhousie University, Halifax, Nova Scotia, Canada (I.W.).

The authors report that they have no conflicts of interest in the authorship and publication of this article. Full ICMJE author disclosure forms are available for this article online, as supplementary material.

Received December 10, 2019; accepted June 5, 2020.

Address correspondence to Ivan Wong, M.D., F.R.C.S.(C.), Dip.SportMed., M.A.C.M., Division of Orthopaedic Surgery, Dalhousie University, 5955 Veterans' Memorial Lane, Halifax, NS,B3H 2E1, Canada.E-mail: iw@ drivanwong.com

(C) 2020 THE AUTHORS. Published by Elsevier Inc. on behalf of the Arthroscopy Association of North America. This is an open access article under the CC BY-NC-ND license (http://creativecommons.org/licenses/by-nc-nd/4.0/). 2666-061X/191531

https://doi.org/10.1016/j.asmr.2020.06.015 with arthroscopic debridement. ${ }^{6,7}$ Other advantages of arthroscopic management include improved visualization, rapid rehabilitation, shorter hospital length of stay, and fewer overall complications. ${ }^{5}$

Septic arthritis in association with an extra-articular abscess is a rare entity. Kim et al. described successful arthroscopic drainage of a psoas abscess concurrent with septic arthritis of the hip in adult patients. ${ }^{8}$ However, to our knowledge, there has been no previous report of arthroscopic debridement of an intrapelvic abscess with concurrent septic hip. In this report, we describe an adult female who received arthroscopic debridement of a septic hip as well as an intrapelvic abscess. The surgical technique for accessing intrapelvic space, pearls, pitfalls, and follow-up of the patient are reported.

\section{Case Report}

A 24-year-old female presented with a 1-month history of atraumatic right groin pain. She had developed nightly pain and sweating during the past week. She had no prior history of hip pathology. She was otherwise healthy, played contact sports and took no medications. There was no history of intravenous drug use, smoking or immune suppression. On examination, she was afebrile, vitally stable and had a noticeable antalgic gait. She preferred her leg in slight flexion and external rotation at rest. Internal rotation of hip was tender and restricted. Bloodwork showed normal serum white cell count (10.4) and elevated C-reactive protein (CRP) levels (117). 

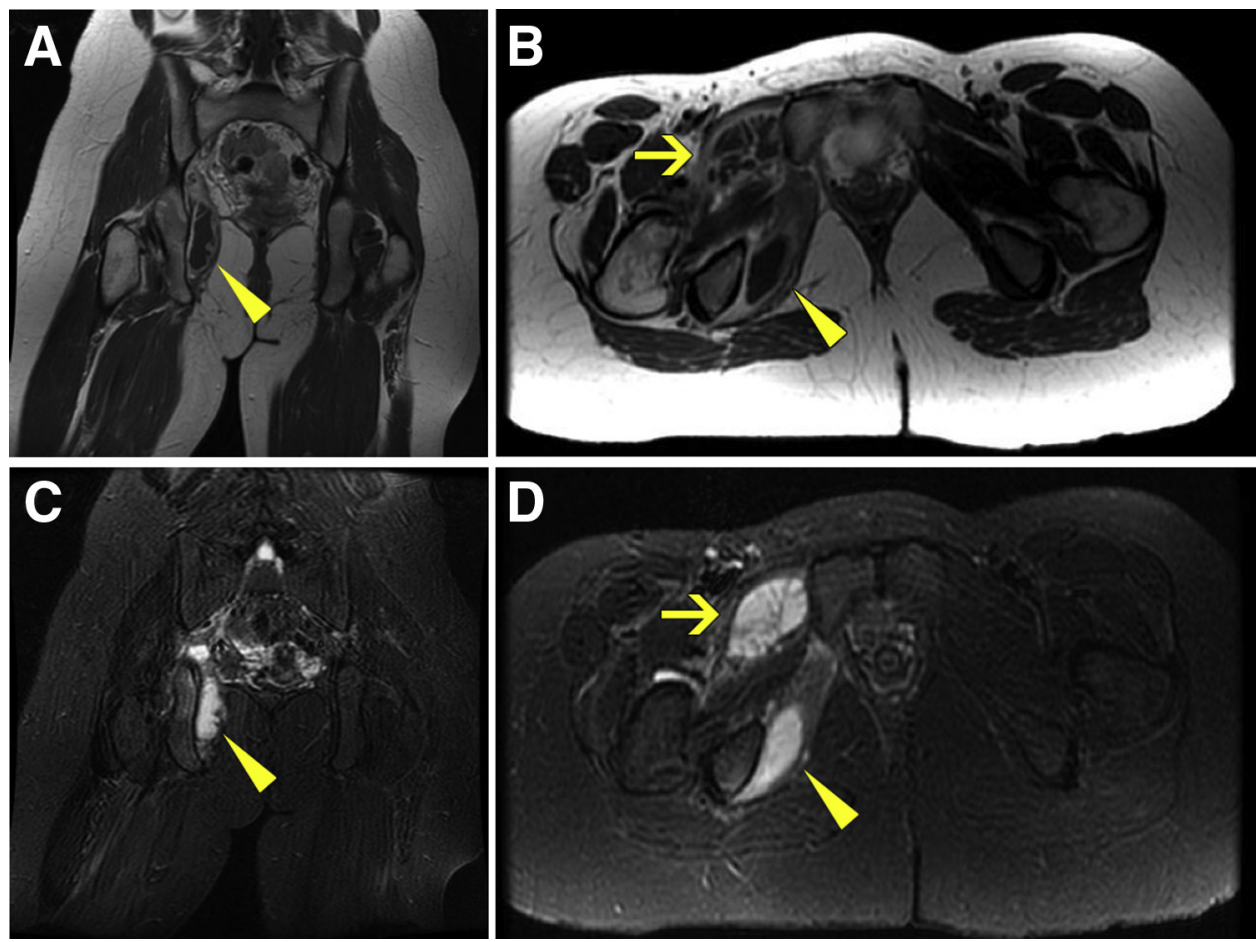

Fig 1. Select MR images of rightsided pelvic abscess prior to arthroscopic debridement. Coronal (A) and axial (B) Tl magnetic resonance images with corresponding coronal $(\mathrm{C})$ and axial (D) of the short T-inversion recovery sequence are shown. An intrapelvic abscess along the lateral border of the right obturator internus measures $6 \times 4 \mathrm{~cm}$ (yellow arrowheads). The abscess continues to the extrapelvic region along the right obturator externus muscle, measuring $5 \times 2$ $\mathrm{cm}$, and communicates with the hip joint (yellow arrows).
A CT scan of her right hip was suggestive of pyomyositis with no evidence of osseous destruction. A magnetic resonance image (MRI) of her pelvis revealed multiple complex, loculated, rim-enhancing fluid collections consistent with abscess within the obturator internus, obturator externus and pectineus muscles (Fig 1). Small right hip joint effusion with synovial enhancement was suggestive of an intra-articular septic process. Antibiotic therapy was withheld until after intraoperative synovial samples had been taken.

\section{Surgical Technique}

General anesthesia was administered with the patient in the supine position. After induction of anesthesia, the patient was placed in the lateral decubitus position with the right hip upward on a standard operating room table. The operative limb was padded and placed flat in traction boot (Hip Positioning System with Active Heel Technology; Smith \& Nephew, Andover, MA). A perineal post was placed on the ischium of the operative side, slightly lateral to midline, and the genitalia were padded. The hip was pretensioned by pushing the traction boot to length, with hip in slight flexion and $30^{\circ}$ of abduction. The hip joint was then distracted by bringing the leg into neutral abduction. Distraction of 1 cm was confirmed on fluoroscopy. The surgical area was prepped and draped in a sterile fashion.

The path of debriding instruments is determined based on preoperative axial MRI images (Fig 2). Standard anterolateral (AL) and distal midanterior portals were placed under fluoroscopic guidance and used as viewing and working portals, respectively. The $\mathrm{AL}$ portal entry was marked anterior to the tip of the greater trochanter, and a long spinal needle was inserted into the hip capsule to estimate its trajectory. The intracapsular position of the needle was confirmed with fluoroscopy by the presence of an air arthrogram. An arthroscopic cannula was then inserted using the

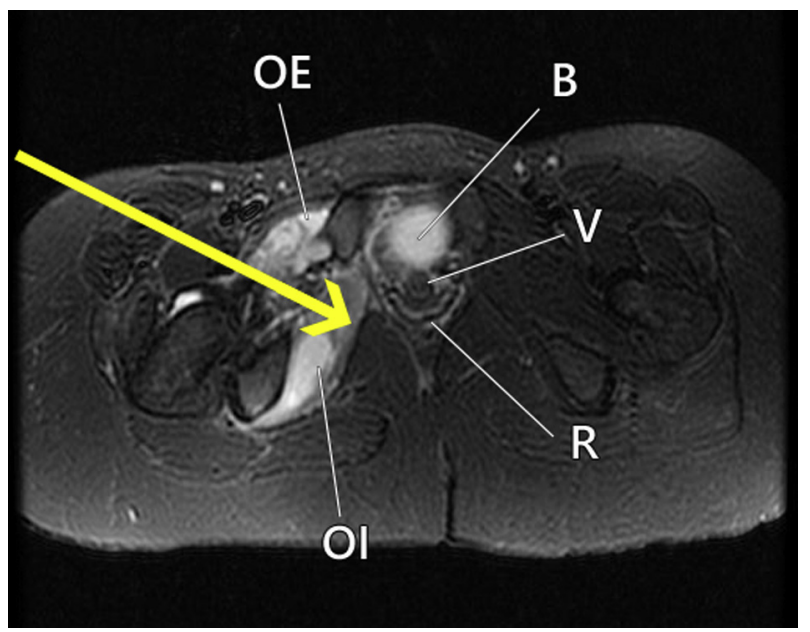

Fig 2. Planned route of intrapelvic arthroscopy. Axial STIR sequence magnetic resonance image of intrapelvic abscess prior to arthroscopic debridement is shown. The arrow signifies the path of the debriding instrument traversing the abscess across the obturator externus (OE) and obturator internus (OI) muscles. Intrapelvic organs including the bladder (B), vaginal canal (V) and rectum (R) are labeled to highlight their proximity. STIR, short T-inversion recovery. 

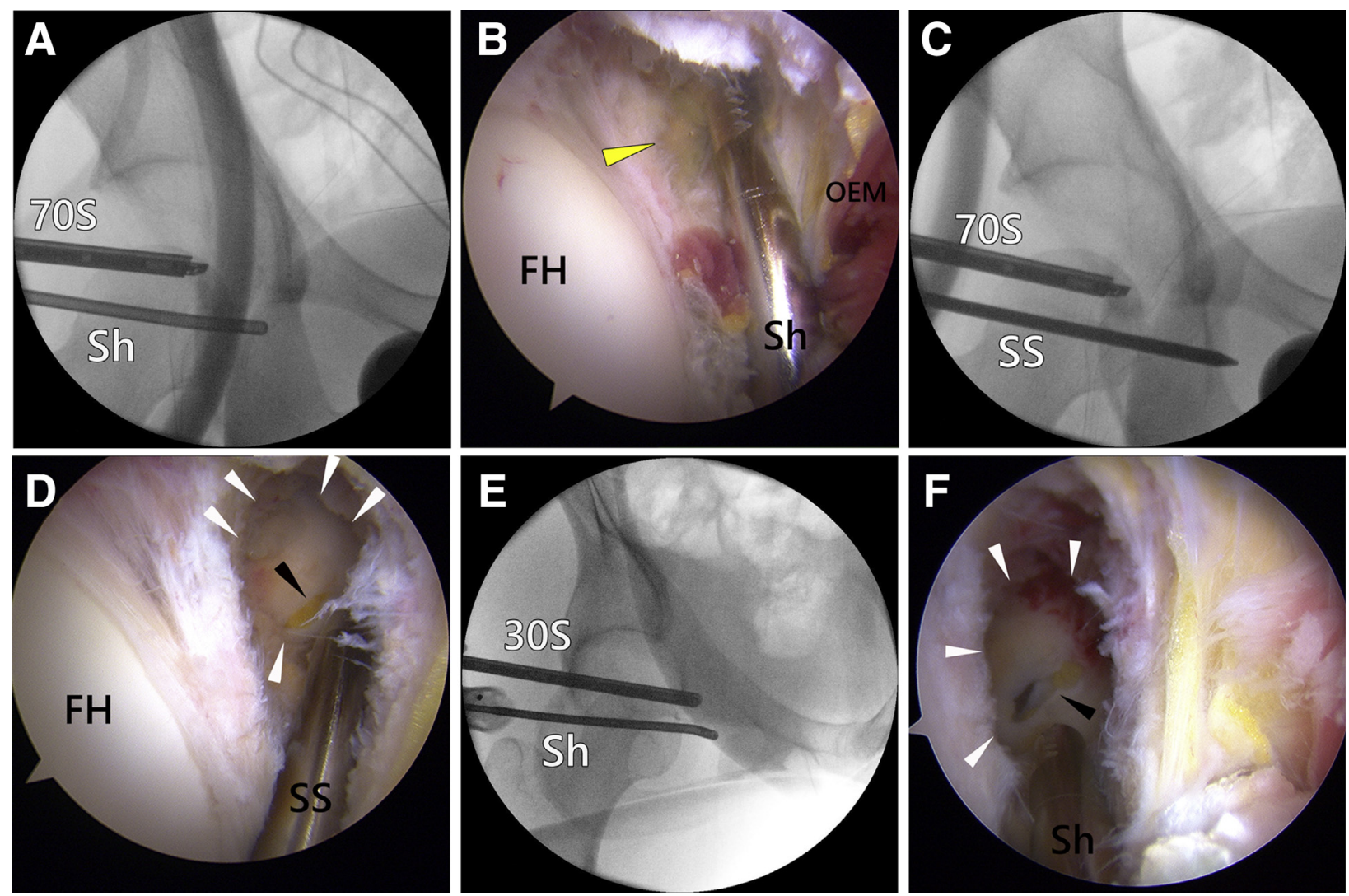

Fig 3. Intraoperative fluroscopy to check instrument placement within the right hip and intrapelvic region. The patient is positioned in a lateral decubitus position with traction applied to the right hip. Standard anterolateral (AL) and distal midanterior (DMA) portals are used as viewing and working portals, respectively. Depth of shaver (Sh) is assessed on anteroposterior fluoroscopy of right hip (A) as it debrides the extrapelvic portion of abscess (B, yellow arrowhead) posterior to the right obturator externus muscle (OEM) and anterior to femoral head (FH). A blunt switching stick (SS) is used to pierce the obturator membrane (white arrowheads outline the obturator membrane; black arrowhead marks the hole) (D). The depth of the SS is monitored by fluoroscopy (C). Switching to a $30^{\circ}$ scope inserted in the AL portal (E, F) allows an expanded view of the obturator membrane and the hole (outlined by white and black arrow heads, respectively).

Seldinger technique over a guidewire. The distal midanterior portal entry was marked $2 \mathrm{~cm}$ distal and medial to the AL portal and completed under direct arthroscopic vision and the use of a guide needle. An interportal capsulotomy was performed with an ablator to facilitate instrumentation. The hip joint was distended with normal saline using an arthroscopy pump set at 40 mmHg (ConMed; Utica, NY).

A $70^{\circ}$ arthroscope was used to perform a diagnostic arthroscopy. Purulence and markedly inflamed synovia, including central foveal tissue, were identified and debrided. Multiple tissue and synovial samples were collected for culture. A thorough synovectomy to healthy bleeding tissue was performed. The capsulotomy was extended medially to the psoas muscle, and the tendinous portions of the psoas were released at the level of the hip joint. Debridement was continued medially and posteriorly to the psoas. The depths at which deeper and more medial structures are expected to be found were measured based on the preoperative
MRI. The obturator membrane can be found posterior and medial to the obturator externus muscle as such debridement is performed along posterior border of the obturator externus muscle (Fig 3A,B). Debridement anterior to the obturator externus muscle risks damaging the obturator neurovascular bundle.

With sufficient debridement, the obturator membrane was encountered and the surrounding tissues were debrided to identify the borders of the membrane. Under direct vision, the central part of membrane was pierced with a blunt switching stick (Fig 3C,D). The hole made in the membrane was enlarged with a shaver. At this point, switching to a $30^{\circ}$ degree scope afforded a better view within the hole (Fig 3E,F). The intrapelvic space could be viewed and accessed through the enlarged hole, and the abscess was decompressed. Obturator internus muscle fibers were visible through the same hole. Accessible regions of the obturator internus were debrided of inflamed tissue; however, debridement was not taken deeper or more medial to 
Table 1. Pearls and Pitfalls of Intrapelvic Arthroscopy Technique

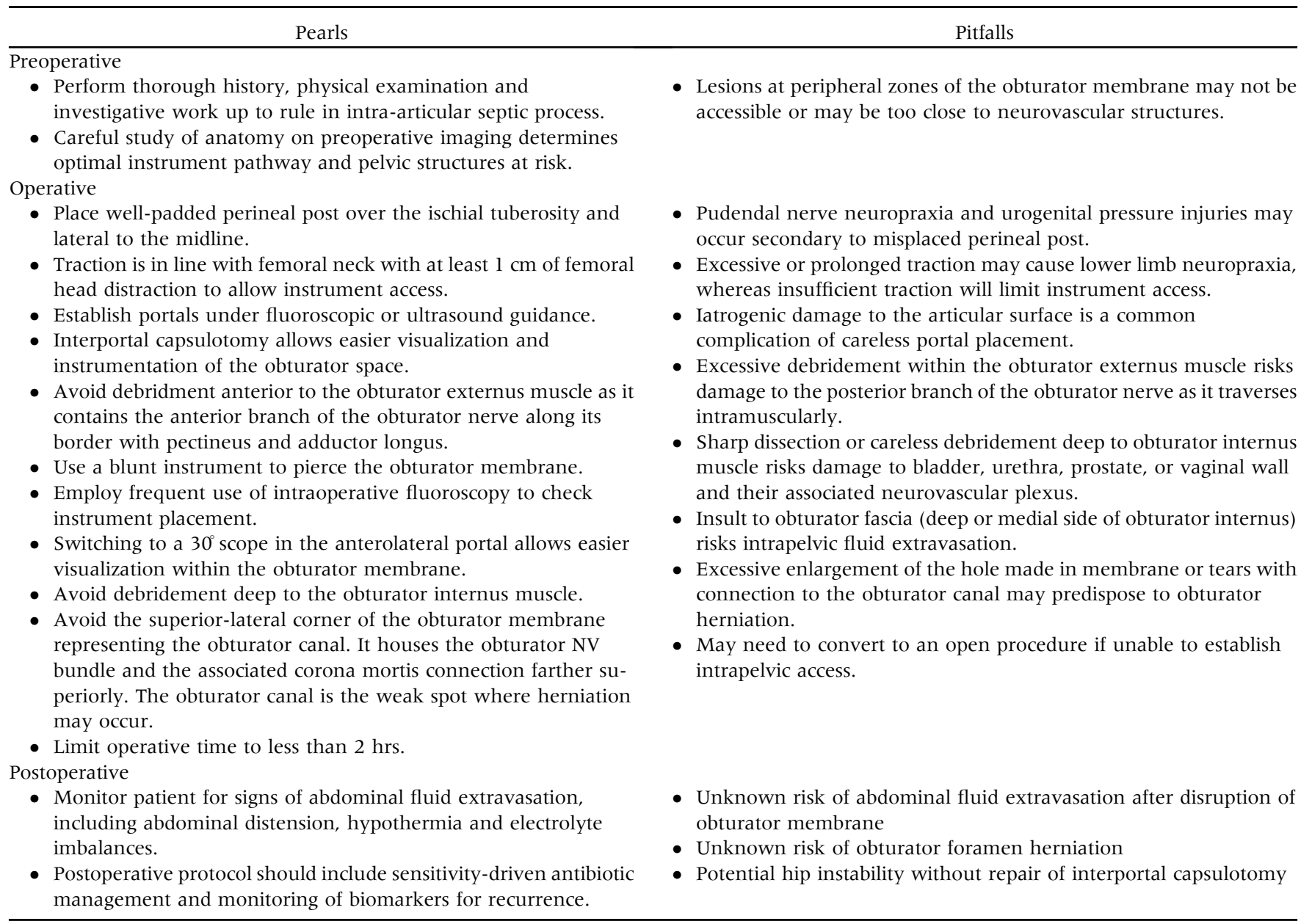

the obturator internus muscle. To access the peripheral zones of the abscess, the shaver was moved within the central hole rather than further enlarging the hole. After satisfactory debridement of the intrapelvic space, the hip joint was copiously irrigated. The capsulotomy was left open to allow further drainage of fluid. No drains were inserted. Pearls and pitfalls of the surgical steps are summarized in Table 1 , and pertinent steps of the procedure are shown in Video 1.

\section{Postoperative Course}

The patient was made to toe-touch with weight bearing and was placed in an abduction brace postoperatively. She remained afebrile and stable, with no
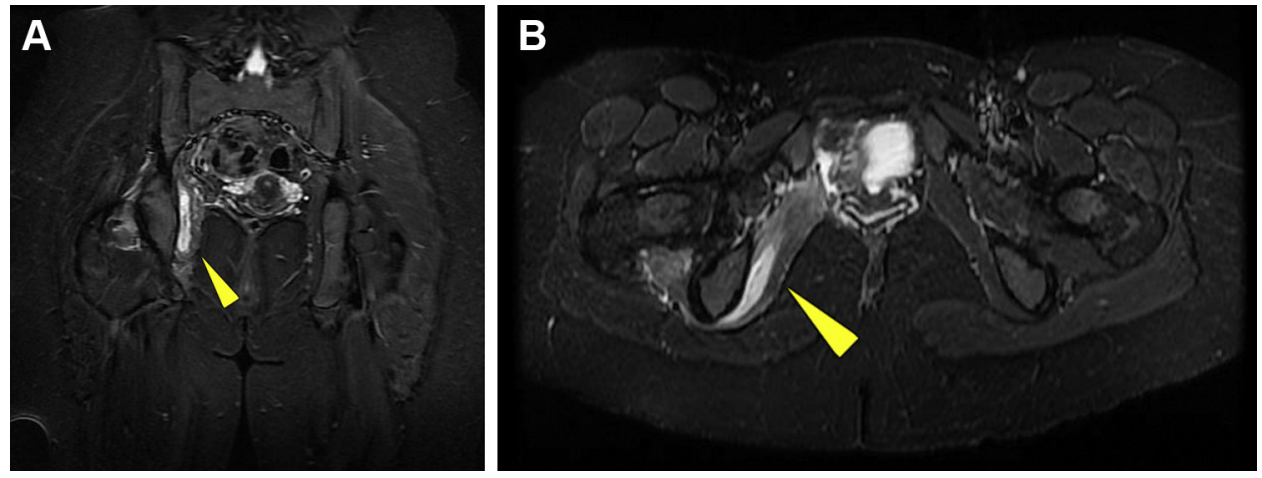

Fig 4. Selected coronal (A) and axial (B) STIR sequence magnetic resonance images of pelvis at 10 months. There has been a recurrence of abscess along the lateral and anterior border of the right obturator internus muscle (yellow arrowhead). Enhancement of right hip intra-articular fluid was suggestive of a reactive or septic process. The patient received an open irrigation and debridement. STIR, short T-inversion recovery. 
Table 2. Advantages and Disadvantages of Arthroscopic Debridement of Septic Hip With Intrapelvic Abscess

Advantages

- Minimally invasive to soft tissue

- Single arthroscopic approach rather than combined open approaches

- Avoids major risks of open arthrotomy, including instability of hip joint and avascular necrosis of the femoral head

- Early postoperative recovery and rehabilitation
Disadvantages

- Limited access, risk of residual abscess, recurrence, and reoperation

- Potential instability with psoas release and interportal capsulotomy

- Unknown risk of postoperative obturator herniation

- Operative learning curve and unclear safe zones need for pressors or stay in the intensive care unit. Immediate postoperatively, her CRP level was 188. Intraoperative cultures did not show a pathogen in any of the 13 samples. The patient received 3 weeks of cefazolin IV after discussion with an infectious-disease specialist. She was discharged home on postoperative day 4.

At the 3-week follow-up, the patient had residual surgical pain. The brace was discontinued and the patient was referred to physiotherapy for range of motion and strengthening. At the 6-week follow-up, the patient was nontender and experienced 4/5 weakness of the right hip flexor. She had good right hip range of motion with $130^{\circ}$ flexion, $45^{\circ}$ abduction, $35^{\circ}$ external rotation, and $15^{\circ}$ internal rotation. CRP levels had normalized to 6.6. At the 6-month follow-up, the patient was completely asymptomatic and regained 5/5 strength of right hip flexors. Her internal rotation improved to $45^{\circ}$ and CRP levels remained normal at 4.4. She scored $88.6 / 100$ on the International Hip Outcome Tool questionnaire.

Unfortunately, the patient had a recurrence 10 months postoperatively. She presented to the emergency department with 1.5 weeks of atraumatic, progressive right buttock pain, antalgic gait and limited right hip range of motion. Her CRP level was 70, and the MRI showed recurrence of the intrapelvic abscess (Fig 4).

\section{Discussion}

In our review of the literature, we found that a protocol for intrapelvic access by arthroscopic means has not been previously published. Of note, Avnieli et al. ${ }^{9}$ described the removal of an intrapelvic obturator ganglion cyst by dilation and fluid decompression through the stalk using principles of l-way valve destruction. It is unclear whether their approach consisted of instrumentation within the pelvis itself. In this article, we have described the technique of establishing intrapelvic arthroscopic access across the obturator membrane. To execute this technique safely, an intimate knowledge of the at-risk structures is required. Therefore, a review of the relevant anatomy is warranted.

The structure most at risk in this technique is the obturator neurovascular bundle. The anatomic course of the obturator neurovasculature has been characterized in multiple cadaveric and radiographic models. ${ }^{10-15}$
The obturator nerve originates from the lumbar plexus, emerges medial to the psoas muscle and courses along the pelvic brim before joining the obturator artery and vein and descending into the obturator canal. The canal is funnel-shaped and is oriented from a smaller intrapelvic opening, which is posterior, lateral and superior, toward a larger extrapelvic opening extending anteriorly, medially and slightly inferiorly. The extrapelvic opening of the canal is composed of the superior pubic ramus superiorly and laterally and the obturator membrane inferiorly and medially. The obturator membrane does not cover the obturator foramen entirely and allows the traversing neurovascular bundle to emerge from the superior lateral corner of the foramen. The obturator nerve divides into anterior and posterior branches. The anterior branch runs anterior to the obturator externus and adductor brevis and posterior to pectineus and adductor longus muscles. The posterior branch dives into the substance of the obturator externus and runs posterior to adductor brevis muscle. The obturator artery branches superficially to the obturator membrane and anastomoses on the interior border of the foramen.

Correlating with our technique, debridement of the abscess within the obturator externus muscle was necessary; however, it risked damage to the posterior branch of the obturator nerve. Risk of damage to the anterior branch of obturator nerve was smaller and was minimized by avoiding debridement anterior or superior to the obturator externus muscle. The next challenge was where to perforate the obturator membrane. We chose a central location to pierce the membrane with a blunt instrument. Perforating the membrane more superiorly would have allowed better access to the retroacetabular abscess. However, this could have compromised the obturator neurovascular bundle at the extrapelvic opening of the obturator canal and, therefore, was avoided.

The perforation within the membrane was not extended to the obturator canal and was not excessively enlarged in order to avoid a possible obturator canal hernia. An obturator hernia is more common in older, multiparous and malnourished females because of the wider pelvis, the loss of supporting connective tissue and the enlarged obturator canal. ${ }^{16,17}$ The first stage of herniation is attributed to weakness of the intrapelvic obturator canal and the protuberance of preperitoneal 
connective tissue into the internal orifice. ${ }^{16}$ Our surgical technique does not affect the internal orifice and specifically avoids extension to or enlargement of the canal itself. Nevertheless, weakening of the obturator membrane may predispose to herniation by causing overall weakness of the connective tissue support.

The depth of intrapelvic instrumentation within the obturator membrane hole was debated, and we elected only to debride the abscess superficial to the obturator internus muscle. Perforation of the obturator internus muscle and medial fascia would have provided a direct path for fluid extravasation. Intra-abdominal fluid extravasation is rare but potentially life-threatening. Female gender, iliopsoas tenotomy, prolonged operative time, and higher fluid pump pressures are identified risk factors for fluid extravasation. ${ }^{18}$ Although no fluid extravasation occurred in our case, there is an inherent risk for this complication in our technique. Therefore, patients should be monitored diligently for signs of fluid extravasation, including abdominal distension, hypothermia, hypotension, and metabolic acidosis. Some authors advocate intraoperative use of a transparent drape over the abdomen so serial abdominal examinations can be performed. ${ }^{18}$

Perhaps the main limitation of this case report is its rarity. This case is the only patient in whom intrapelvic arthroscopy was attempted at our center, and we cannot generalize our success or complication rate. The patient's age, gender and lack of comorbidities certainly favored a positive outcome and mitigated the immediate perioperative adverse events. In fact, the patient did not possess any risk factors to instigate a septic process, and the cause of the primary abscess is unknown. The major complication was the recurrence of abscess and the open debridement. The recurrence of the abscess may be attributed to the surgical technique or to a secondary septic process. In the case of recurrence due to incomplete debridement, we found it unusual that the patient was clinically asymptomatic and had normal CRP levels (4.4) at 6 months postoperation. Advanced imaging would have proved helpful in identifying the persistence of abscess; however, it was deferred due to absence of the patient's symptoms. Ultimately, the causes of the primary and the recurrent abscess remain unclear to us.

Hip arthroscopy is a rapidly expanding surgical field owing to its minimally invasive approach and the quick rehabilitation afterward. Our technique introduces a new area of arthroscopic interventions. We have described how to access lesions deep to the obturator membrane safely. A summary of the advantages and disadvantages of this technique is provided in Table 2 . Our technique can be further developed by exploring additional portals and defining size- and locationspecific indications for intrapelvic access. In the experience of the senior author (IHW), central, medial and inferior retro-obturator lesions may be more amenable to intrapelvic technique because they are farthest away from vulnerable anatomic structures.

\section{Conclusion}

Intrapelvic obturator space can be accessed safely through hip arthroscopy and represents a new area for arthroscopic interventions. Careful patient selection and preoperative planning are recommended.

\section{References}

1. McCarthy JJ, Dormans JP, Kozin SH, Pizzutillo PD. Musculoskeletal infections in children: Basic treatment principles and recent advancements. Instr Course Lect 2005;54:515-528.

2. Sucato DJ, Schwend RM, Gillespie R. Septic arthritis of the hip in children. J Am Acad Orthop Surg 1997;5:249-260.

3. Lee Y-K, Park K-S, Ha Y-C, Koo K-H. Arthroscopic treatment for acute septic arthritis of the hip joint in adults. Knee Surg Sports Traumatol Arthrosc 2014;22: 942-945.

4. Kim S-J, Choi N-H, Ko S-H, et al. Arthroscopic treatment of septic arthritis of the hip. Clin Orthop Relat Res 2003;407: 211-214.

5. de SA D, Cargnelli S, Catapano M, et al. Efficacy of hip arthroscopy for the management of septic arthritis: A systematic review. Arthroscopy 2015;31:1358-1370.

6. El-Sayed AMM. Treatment of early septic arthritis of the hip in children: Comparison of results of open arthrotomy versus arthroscopic drainage. J Child Orthop 2008;2: 229-237.

7. Schröder JH, Krüger D, Perka C, Hufeland M. Arthroscopic treatment for primary septic arthritis of the hip in adults. Adv Orthop 2016 [accessed Sep 30, 2019]. Available at, https://Www.ncbi.nlm.nih.gov/pmc/articles/PMC5069 $383 /$.

8. Kim C-H, Aditya K, Lee S-J, et al. Arthroscopic treatment of psoas abscess concurrent with septic arthritis of the hip joint. Hip Int 2018;28:336-340.

9. Bachar Avnieli I, Amar E, Efrima B, et al. Hip arthroscopy as a treatment for obturator neuropathy secondary to intra-pelvic ganglion: A case report. J Hip Preserv Surg 2018;5:319-322.

10. Kendir S, Akkaya T, Comert A, et al. The location of the obturator nerve: A three-dimensional description of the obturator canal. Surg Radiol Anat 2008;30:495-501.

11. Kumka M. Critical sites of entrapment of the posterior division of the obturator nerve: Anatomical considerations. J Can Chiropr Assoc 2010;54:33-42.

12. Jo SY, Chang JC, Bae HG, et al. A morphometric study of the obturator nerve around the obturator foramen. J Korean Neurosurg Soc 2016;59:282-286.

13. Petchprapa CN, Rosenberg ZS, Sconfienza LM, et al. MR imaging of entrapment neuropathies of the lower extremity. Part 1: The pelvis and hip. Radiographics 2010;30: 983-1000.

14. Whiteside JL, Walters MD. Anatomy of the obturator region: Relations to a trans-obturator sling. Int Urogynecol $J$ Pelvic Floor Dysfunct 2004;15:223-226. 
15. Bonnet P, Waltregny D, Reul O, de Leval J. Transobturator vaginal tape inside out for the surgical treatment of female stress urinary incontinence: Anatomical considerations. J Urol 2005;173:1223-1228.

16. Petrie A, Tubbs RS, Matusz P, et al. Obturator hernia: Anatomy, embryology, diagnosis, and treatment. Clin Anat $2011 ; 24: 562-569$.
17. Stamatiou D, Skandalakis LJ, Zoras O, Mirilas P. Obturator hernia revisited: Surgical anatomy, embryology, diagnosis, and technique of repair. Am Surg 2011;77: 1147-1157.

18. Ekhtiari S, Haldane CE, de Sa D, et al. Fluid extravasation in hip arthroscopy: A systematic review. Arthroscopy 2017;33:873-880. 